\title{
Healthy aging segments in older Irish adults
}

\author{
S.N. McCarthy ${ }^{1}$, M. McCarthy ${ }^{2}$, D.I. O'Connor ${ }^{3}$ and S McGrath ${ }^{1,3}$ \\ ${ }^{1}$ Department of Agrifood Business, Teagasc Food Research Centre, Ashtown, Dublin 15, ${ }^{2}$ Department of Food Business \\ \& Development, University College Cork and ${ }^{3}$ School of Food Science and Environmental Health, Dublin Institute of \\ Technology
}

In almost all countries across the globe the proportion of the population aged over 60 years is expanding at a rate faster than all other age groups. The World Health Organisation has forecast that $25 \%$ of the European population will be aged 65 and older by 2050 (WHO, 2014). It is of particular importance that this cohort of the population adopt a healthy diet and lifestyle to prevent malnutrition and its associated consequences such as sacropenia and cognitive decline. However, this older group of adults are not homogenous in their food choice attitudes or behaviour and therefore require targeted public health promotion campaigns and policies to promote healthy aging.

The aim of this study was to determine if healthy aging segments of older adults ( $>50$ years) could be identified based on food choice attitudes, motives and behaviours. Data on diet, lifestyle, and food choice attitudes in adults aged greater than 50 years from the National Adult Nutrition Survey were used.

Dietary intakes and attitudes were clustered using K-means cluster analysis to generate consumer segments. Differences in mean attitudinal scores across the three resultant clusters were calculated to provide additional characteristics and profiling of each cluster.

\begin{tabular}{|c|c|c|c|c|c|c|c|c|}
\hline Cluster name & Men & Women & Smoker & Normal BMI $\%$ & EnFat & EnProtein & EnCho & Alcohol units/wk \\
\hline Sensible Lifestyle & 41 & 59 & 6 & 32 & 31 & 18 & 51 & 5 \\
\hline Poor Lifestyle & 46 & 54 & 11 & 20 & 37 & 18 & 45 & 4 \\
\hline Risky Lifestyle & 69 & 31 & 17 & 15 & 33 & 17 & 50 & 24 \\
\hline
\end{tabular}

Cluster analysis generated three segments as illustrated in the table above. Membership of the 'Sensible Lifestyle' segment was associated with following a healthy diet, being female, having excellent self-rated health and less time spent watching television. The average age was 60 years and had a high prevalence of normal weight subjects. The second segment 'Poor Lifestyle' was characterised by poorer quality diet, low levels of physical activity and lower educational attainment. More than $20 \%$ of this group rated their health as less than good. The most outstanding characteristic of the third segment 'Risky Lifestyle' was the high level of alcohol consumption, with an average intake of 24 units per week. This group had low fruit and vegetable intake and was associated with being male and smoker.

Targeted and specific approaches are required to maintain the positive health trajectory of the 'Sensible Lifestyle' segment. Public health campaigns should aim to address and highlight the high alcohol consumption in the 'Risky Lifestyle' particularly men aged 50 60 years and increase the awareness of the long term ill-effects. However, the largest proportion of the population in the 'Poor Lifestyle' segment would benefit from assistance in making healthier and better informed foods choices. In the attitudinal analysis they acknowledge the importance of health and nutrition in making food choices and yet it does not translate into dietary behaviour. They are also the most vulnerable group with the highest proportion of adults aged over 70 . This group would benefit from targeted new product development that takes into consideration age related health issues such as decreased sensory perceptions and specific requirements for many nutrients in an attempt to positively influence eating behaviour and nutrient status in this segment.

\section{Acknowledgements}

The funding to carry out the NANS survey and subsequent secondary analysis was provided under the FIRM funding programme of the Department of Agriculture, Food and the Marine. 